\title{
Designing Digital Engagements: Approaches to creative practice and adaptable programming for archaeological visualisation
}

\author{
Alice Watterson \\ 3DVisLab, University of Dundee \\ 13 Perth Rd, Dundee DD1 4HT, UK \\ a.z.watterson@dundee.ac.uk
}

\author{
John Anderson \\ 3DVisLab, University of Dundee \\ 13 Perth Rd, Dundee DD1 4HT, UK \\ j.r.anderson@dundee.ac.uk
}

\author{
Kieran Baxter \\ 3DVisLab, University of Dundee \\ 13 Perth Rd, Dundee DD1 4HT, UK \\ k.w.baxter@dundee.ac.uk
}

\begin{abstract}
The processes of archaeological visualisation exist at the intersection of art practice and archaeological interpretation, often involving complex negotiations between stakeholders and practitioners. This paper reflects upon the authors' experiences developing interactive mixed media content for public outreach from two case study archaeological excavations: the SERF Hillforts Project in Strathearn, Scotland and the Nunalleq Archaeology Project in southwest Alaska. Each presented unique challenges in the integration of layered multivocal narratives in the context of ongoing archaeological excavations. This included evolving scientific interpretations, co-design with stakeholders and a cycle of feedback. Creative design and software development were a core part of the collaborative process that resulted in these interactive digital interfaces. Here we explore how collaborative creative practice influenced the design choices that were made and the programming paradigms that were used.
\end{abstract}

Archaeology. Heritage. Public outreach. Community archaeology. Interactive visualisation. Co-design.

\section{INTRODUCTION}

This paper aims to provide a reflexive insight into the collaborative processes involved in the production of interactive media for outreach and engagement in the context of two archaeological excavation projects: the SERF Hillforts Digital Project in Strathearn, Scotland, and the Nunalleq Educational Resource in southwest Alaska.

Through discussion of the design practice, programming methodology and implementation we will explore how and why creative design decisions were made based on the context of the specific user group, collaborator and stakeholder requirements while giving consideration to the challenges of archaeological interpretation and visualisation with regard to transparency and modes of engagement.

\section{AIMS AND METHODOLOGY}

The two case studies discussed here share the challenges of multivocal representation and codesign with stakeholders in the context of creating engaging public outreach. The design and software development approach adopted by these projects took into account the requirements that emerged from both creative practice and stakeholder discussion. As such, this work resulted in an evolving framework for the exploration of digital curation and interactive user-experiences.

Through both projects our aim was to facilitate an inclusive design process that layers narratives and meaningfully engages with stakeholders, scientists and communities to create tangible heritage outreach material for general audiences by asking the following questions:

- How do we generate interactive outputs, which open up the processes of archaeological interpretation to the public by establishing a clear visual language?

- How might we integrate multi-vocality and their context into a coherent narrative?

- How do we cultivate a collaborative environment for co-design?

\section{THE SERF HILLFORTS PROJECT}

The SERF Hillforts digital outreach project was initiated following a series of discussions between the 3DVisLab team and the SERF hillforts project director (Dr Tessa Poller), reflecting on the fluidity of her interpretive process over a 10-year period of 
excavations of multiple Iron-Age hillfort sites in the Strathearn region of Scotland. As such, this case study adopted a practical approach to current issues of representation and audience engagement within archaeology through the development of an interactive online

resource (www.seriousanimation.com/hillforts), which explores the ways in which archaeologists craft their interpretive narratives on-site.

Archaeologists have long problematised the role of the computer-generated reconstruction image as everything from seductively subjective in its aesthetic (Turkle 2009 and Richards 2005), to technologically superior when compared to the traditional 'artist's impression' painting and therefore more scientifically cohesive (Huggett 2004; Zubrow 2006). As a result the field still grapples with a series of deeply embedded broadbrush expectations about what these types of visualisations can and should do, regardless of their context (Watterson 2015). Consequently, the SERF Hillforts interface aimed to use visualisation as a means to better reflect the fluidity of the interpretive process and engage audiences more meaningfully with the challenges that the representation of evidence can pose to archaeologists. Using digital reconstruction, interaction and audio narratives the team explored the possibilities for digitally curated content, which more authentically represents the multi-layered, multivocal and often ambiguous processes involved in archaeological interpretation.
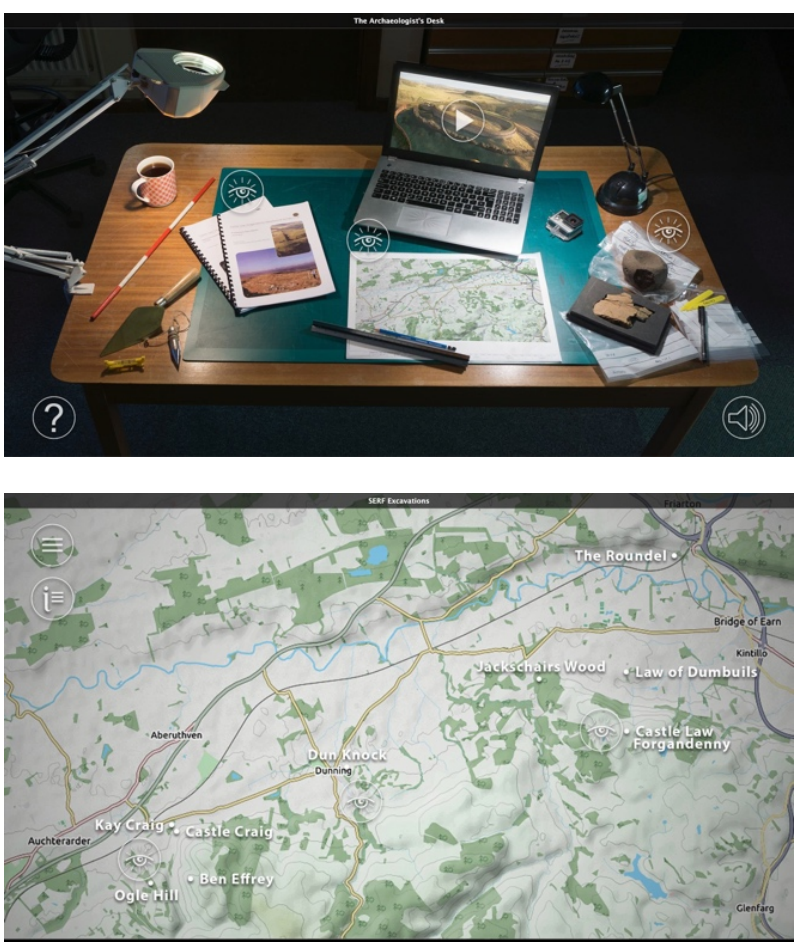

Figure 1: The Archaeologist's Desk main menu page and the interactive SERF Excavations map.
The SERF Hillforts Project was conceived from the outset as an interactive multimedia work to be made accessible online. A non-interactive prototype was developed early on in the design process in the form of a short video demonstrating how each of the components would work and how they would look. The prototype included interactive multimedia aspects that would carry through to the final product, such as the graphic metaphors of the Archaeologist's Desk and the interactive SERF Excavations Map (Figure 1). Navigation icons and other screen-based controls were defined, including a slider that was designed to provide the capability to "fade through" alternate visual content. This was conceived as a visual and interactive tool for representing the layers of interpretation that were at the heart of the interface design.

The final design included both linear and non-linear narrative pathways, some with branching navigational structures. Whilst the prototype did not attempt to depict all of the content and interactions planned for the final product it nonetheless laid the foundations for the visual layout and intrinsic structure to be carried forward to an interactive proof-of-concept. One of these narrative pathways involved a fly-in from the broader landscape of the Strathearn region to the detail of the individual hillfort sites, as selected by the user. A topographical map with the hillforts marked among features of the modern-day landscape was used to represent the wider valley (Figure 1 ). While this cartographic representation is a visual abstraction from landscape as known from the ground it serves to give an impression of the geographical contiguity of the valley along which the sites are distributed, as well as the landmarks that might be familiar to local inhabitants. Animated fly-in videos, based on oblique aerial photogrammetry, then bridged the gap between the map view and a more grounded perspective (see Baxter 2014). The narrative was structured in this way in order to visually connect the sites to a sense of the landscape, both abstracted and grounded, as a common platform for understanding shared by specialists and nonspecialists alike. This laid the context for the other key component of the narrative structure, which led the viewer through the layers of interpretation of the detail of each hillfort, in part using speculative reconstruction.

Being transparent about the nature of interpretation, and the ways in which we construct interpretive imagery, is an important consideration when communicating archaeological practice to audiences (Watterson 2015). In archaeology, particularly in the context of reconstruction work, the process of creating a single image encourages a somewhat problematic, conclusive result, which does not best reflect the processes of archaeological interpretation. More often than not 
we are left with more questions, varying hypotheses and multiple unknowns. The SERF Hillforts Project was different from a conventional reconstruction brief because rather than asking simply for a reconstruction of a particular hillfort, the brief asked how we might better visualise the archaeologist's interpretive process. This was addressed through the interface design in a number of key ways, which layered interpretive narratives within the visualisations. Firstly, reconstructions of Castle Law Forgandenny included multiple cut-away options for the wall construction controlled by a slider bar (Figure 2). Secondly, a pinboard interface was conceived for Ogle Hill, which combined 'trench-edge' interpretive narratives filmed in the field with animated annotations over excavation photographs (Figure 3). Finally, conversations and narrations concerning details on the interpretive process were included throughout the interface, accessed via icons on each page. This allowed users to hear discussions between various specialists relating to how the excavations were planned, decisions made and interpretations reached.

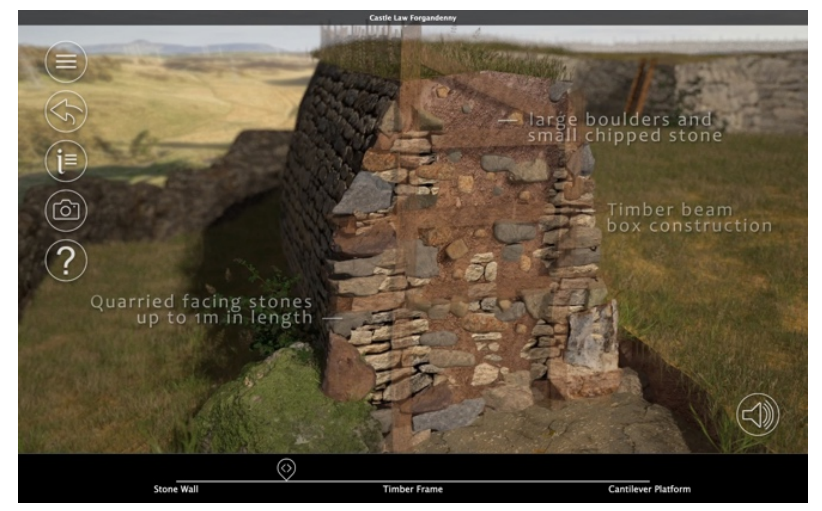

Figure 2: The cut-away reconstruction of the wall at Castle Law Forgandenny.

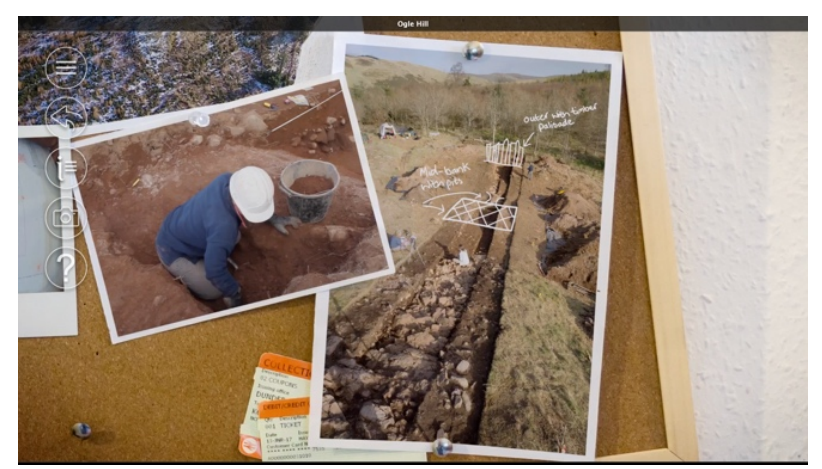

Figure 3: The pinboard interface showing a detail of animated annotations of excavation photographs.

The project demonstrates the possibilities for bringing together a range of visual digital media (photogrammetry, aerial photography, survey data, $3 \mathrm{D}$ reconstruction, film-making and digitised site reports) to open up the processes behind the excavation and interpretation to a general audience and act as an interactive archive now that the excavations have concluded. Audio narratives paired with the visual design of the archaeologist's and specialist's desk backdrops all came together to invite the audience behind the scenes as much as possible. The idea behind this was to recognise the importance of including "paradata" (see Denard 2012) in a way that is relevant and meaningful to non-specialists.

\section{THE NUNALLEQ RESOURCE}

The Nunalleq Archaeology Project saw the codesign of a digital educational resource (www.seriousanimation.com/nunalleq), which tells the story of the excavations of a Yup'ik sod house near the village of Quinhagak, Alaska. The resource, Nunalleq: Stories from the Village of our Ancestors, was co-designed between the village board (Qanirtuuq Inc., the ANCSA village corporation in Quinhagak), local community in Quinhagak, archaeologists from the University of Aberdeen and the production team at the 3DVisLab, University of Dundee. The aim of the resource was to communicate the archaeological findings with Yup'ik schoolchildren (7-15 age range) as the core user-group and in so doing support the local community's goals to save, preserve and share the archaeological material for future generations. The Quinhagak village board asserted that the resource should be based in digital media to make it more appealing to the younger generation, continuing the growing trend of digital platforms for cultural engagement amongst Arctic regions (for example the Thule house VR experience and Journey to Kitigaaryuk covered in Levy and Dawson 2014 and the popular Iñupiaq platform game Never Alone 2014 (Schlag 2018). Fostering the culture of traditional knowledgeexchange that the excavations had established over the course of the project (Fienup-Riordan, Rearden \& Knecht 2015; Hillerdal, Knecht \& Jones 2019), the underlying framework design for the interface made space for traditional knowledge and contemporary engagements alongside scientific interpretation, by facilitating a multi-vocal narrative for the archaeological site.

In the context of community co-design the methodology adopted by the production team (i.e. designer, artist and programmer) for this project allowed for improvisation and flexibility in order to adaptively respond to the evolving feedback, suggestions and ambitions of the village, as well as the developing interpretations emerging from an ongoing excavation. This responsive approach was essential in the context of indigenous representation where a sense of stewardship and identity goes deeper than ownership of the material 
remains (Hillerdal 2017). Native communities taking an active role in the research and communication effort is a crucial part of addressing a history of problematic one-sided arrangements where scientists take rather than share (Dawson 2001; Clifford 2013). Incorporating these considerations and responding to the feedback from community members as part of an iterative methodology meant that the visual material put Yup'ik faces, voices, curation and philosophies at the forefront of this outreach material. This is in line with the mission statement of the Nunalleq Project as a whole: to engage in a socially aware, relevant and involved archaeology that has a bearing for people outside of academia (Hillerdal 2018). It also resonates with Smith's (2012) assertion that people should recognise themselves and have an active role in representations of their culture.

During the early stages of the project the team prepared for the upcoming 2017 field season by developing pre-production visual material based on the initial brief which could be used as a foundation for workshopping these concepts with the local community in Quinhagak and the extended archaeological crew. This pre-production stage used mood and design boards with environment sketches suggesting atmosphere and early stage character design together with suggested modes for possible interactions and content. This rough conceptual material aimed to map out enough of a basic design for the interface and an indication of loose themes for the content so that collaborators had something to immediately feedback on. However, this material was presented as a preliminary outline, allowing space for others to contribute ideas and collaboratively build towards a final outcome.

The resulting interface for the Nunalleq resource (Figure 4) was shaped by key requirements identified by the community, which prioritised a hands-on approach to representing their heritage. This engaged approach aimed to bring objects out of the conventional museum cabinet and into the living Yup'ik culture. Furthermore, traditional approaches to inter-generational teaching using spoken word (Kawagley 1990), rather than written text, were favoured, acknowledging and representing the enduring role of Elder knowledge in Yup'ik culture. Consequently, the main interface presented the artefacts within a speculative reconstruction of their environment and invited users to discover the objects and their interpretations by exploration rather than an ordered typology. Users can explore a reconstruction of the sod house from various vantage points and click on a selection of objects within the scene (Fig 6.) to bring up a 3D viewer with the relevant artefacts. Soundbites can be activated by clicking on icons, which are representative of the source of information: male and female elders and community members, archaeologists and young people.

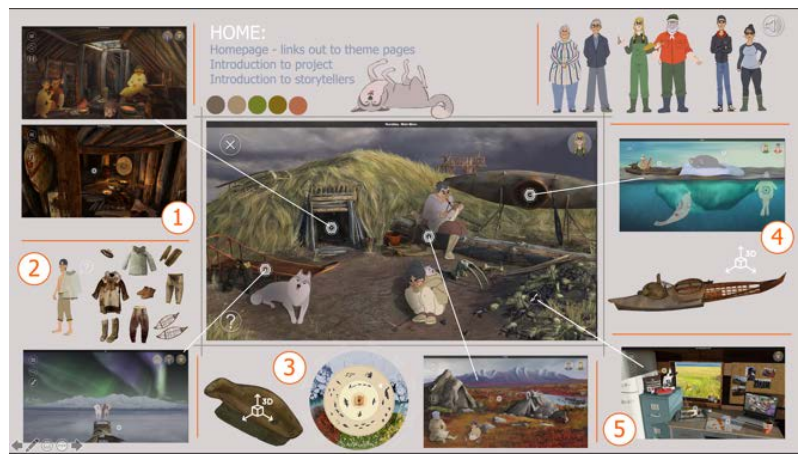

Figure 4: Overview of the educational resource showing content branching from the homepage.

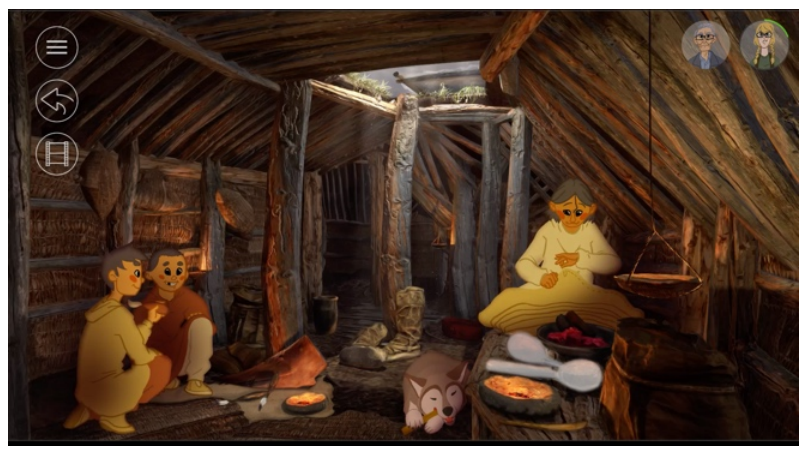

Figure 5: Exploring the sod house and clicking on artefacts (note highlighted objects on lower right).

Each scene the user encounters, from the house to various locations in the wider landscape, was rendered as atmospheric looped animations with characters engaged in activities reinforcing the narrative, and using artefacts excavated from Nunalleq. Digital reconstructions in archaeology can often appear sterile; empty structures detached from the chaos and mess of daily life, which serve to visualise a space rather than an experience of a place and time (Earl 2004). This tendency for sanitised representations can be detrimental to the process of connecting with the intended audience, resulting in images that can be alienating and difficult to engage with (Jeffrey 2015, p.145). Smith and Campbell (2016) argue that to ignore the emotional resonance of heritage is to ignore meaningful engagements with the ways in which people in the present use the past as a contemporary cultural resource (Levy \& Dawson 2014). This is relevant to the ways in which the inhabitants Quinhagak are using their cultural heritage to re-interpret their contemporary Yup'ik cultural identity (Watterson \& Hillerdal, in review). 


\section{DISCUSSION}

Evaluating this work is challenging as presently in archaeology there are no defined methodologies for the creation of visualisation works which actively encourage and embrace the creative process. Law (2004) observes that typically the social sciences have difficulty dealing with processes, which involve 'messy thinking' because clear descriptions are not always successful if what they are describing is not always coherent. As Watterson (2015) has previously observed, despite the efforts of practitioners who advocate transparency and documentation of the visualisation process by means of metadata and paradata (Denard 2012) it remains difficult to systematically ascertain the 'success' of a particular visualisation as the subjective aspects of its character often make it resistant to conventional evaluation techniques prevalent in science (Earl 2004).

Ultimately, the design processes underpinning the creation of these interactive interfaces was influenced by the nature of archaeological practice and interpretation, which can often be at odds with itself. Archaeology can be defined as the study of material culture; where material is measurable, functional and dateable but culture is less so, pertaining more towards lived-experience and contextual worldview. As archaeologists and science-communicators it can be challenging to make our research engaging and relevant to the broader communities we work within. But nonspecialist audiences often relate to the past in more personal and emotive ways (Levy \& Dawson 2014). In this way these projects begin to better embody the complex character of archaeological interpretation, while working collaboratively ensures that we are actively working towards making archaeology relevant for people today. However, working collaboratively on community projects is not always straightforward, and being inclusive means more than simply inviting everyone to participate. It requires a willingness to adapt and modify the approach to include people in a way that works for the collaborators and affords a feeling of ownership over the final collective results (Watterson \& Hillerdal, in review).

This need for adaptability is also true of the software design processes used in realising the outcomes. Although the tangible outputs of this research are software-based systems, the authors would stress that we do not see the process of realising them as software development in the conventional sense. In the past creating software has often been undertaken in discrete, sequential steps that had names such as "requirements", "analysis" and "program design" (Royce 1970). Such a traditional approach, characterised by an imperative to manage project scope through early negotiation and locking in of functional requirements is not always conducive to a positive experience of creative collaboration. More recent software development styles such as Agile (Beck et al. 2001) that advocate iteration over traditional "up front" design provide better conceptual frameworks for supporting collaborative practice but nonetheless remain specification-driven and are perhaps best applied within the domain of software development rather than among creative collaborators from diverse disciplines.

In contrast to traditional methodologies, the authors' goal has been to approach software development so that the programmer's role becomes actively to open up scope for the inclusion and representation of evolving narratives and to seek to avoid arbitrary technical constraints, especially in the formative stages of co-design. In pursuing this approach the aim has been to identify appropriate technologies that may help to evolve a programming paradigm that supports the authors' visually-led creative process and provides rapidity of feedback in the coding and evaluation cycle. Philosophically such an approach has some parallels to the recent phenomenon of Live Coding which has brought immediacy and performativity to programming and supports an "artistic compulsion to change one's mind mid-progress" (Collins 2011).

The representation of both knowledge and narrative within these projects is multimodal by design and consequently there is an equal reliance on the modalities of text, animation, video, audio and 3D experiences. This has led the authors to consider that interactive multimedia practice has a methodological relevance to this problem domain. Interactive multimedia has always been an inherently collaborative enterprise and a product of the intersection of art practice and computing technology (Elsom-Cook 2001, p.268). Interactive multimedia products have often been realised using proprietary authoring systems. However, as Butlerman and Hardman (2005) discuss, such systems attempt to fit the authoring process to defined workflow archetypes (tree structures, graphs, timelines and program code), which, in the authors' view, limit their adaptability to our purpose. Another significant disadvantage of these systems is that they encapsulate knowledge in closed and opaque data formats.

Technology choice has been a critical factor in whether, and to what extent, a fluid and adaptive programming paradigm that is supportive of the codesign process might be achieved. The expressive potential of the web technologies upon which these outcomes were based afforded opportunities to create engaging software experiences without recourse to traditional software or multimedia development methodologies. Enacting a "minimal 
coding" strategy, facilitated by critical technology selection, contributed to the co-design process and collaborative approach without drawing focus away to implementation issues.

\section{CONCLUSION}

The case studies discussed here provide a reflexive tool for us as practitioners to better articulate how the methods and media we use frame the knowledge we create. Through the processes of collaboration and co-design these projects suggest a methodology for the production of interpretive archaeological material, which explores transparency through establishing a critical approach to visual language. A collaborative environment was upheld throughout each project by establishing a responsive and adaptable working methodology relevant to the production context. This design process was supported by a content-orientated coding strategy and careful consideration of the technologies used. Ultimately, this paper demonstrates a practice-based approach to exploring the complex negotiations between practitioners and stakeholders in a bid to bring greater clarity to the processes behind the creation of interpretive archaeological imagery through interactive media.

\section{ACKNOWLEDGEMENTS}

The SERF Hillforts Digital Project was funded by Historic Environment Scotland, while the Nunalleq Educational resource was funded by the Arts and Humanities Research Council through grants (AH/K006029/1) and (AH/R014523/1), a University of Aberdeen IKEC Award with additional support for travel and subsistence from the University of Dundee, DJCAD Research Committee RS2 funding.

Thank you to the many people who contributed their support, knowledge, feedback, voices and faces throughout these projects, this includes members of the local communities in Dunning and Quinhagak. We would also like to acknowledge the hard work of other team members for both projects including Tessa Poller, Cathy Maclver and Kieran Duncan for SERF and for Nunalleq, Charlotta Hillerdal, Rick Kneckt, Warren Jones, Grace Hill, Dora Strunk, Pauline Matthew, Crystal Carter and Tom Paxton.

\section{REFERENCES}

Baxter, K. (2014) Grounding the Aerial: The Observer's View in Digital Visualisation for Built Heritage. Electronic Visualisation and the Arts: proceedings of the EVA 2014 conference. London, UK, 8-10 July 2014, pp.163-170. BCS, London.

Beck, K., Beedle, M., van Bennekum, A., Cockburn, Cunningham, W., A., Fowler, M., Grenning, J., Highsmith, J., Hunt, A., Jeffries, R., Kern, J., Marick, B., Martin, R., Mellor, S., Schwaber, K., Sutherland, J. and Thomas D. (2001) Manifesto for Agile Software Development. http://agilemanifesto.orgl (retrieved 10 Mar 2020).

Clifford, J. (2013) Returns: Becoming Indigenous in the Twenty-First Century. Harvard University Press, Cambridge, MA.

Collins, N. (2011) Live Coding of Consequence. Leonardo, 44(3), pp.207-211.

Dawson, R,A,O. (2001) Bridging Traditions and Science. In A. Crowell, A. Steffian and G. Pullar (eds.), Looking Both Ways: Heritage and Identity of the Alutiiq People. University of Alaska Press, Fairbanks.

Denard, H. (2012) A New introduction to the London Charter. In A. Bentkowska-Kafel, H. Denard, and D. Baker (eds.) Paradata and Transparency in Virtual Heritage. Ashgate, Farnham.

Earl, G. P. (2004) Video Killed Engaging VR? Computer Visualisations on the TV Screen. In Smiles, S. and Moser, S. (eds.) Envisioning the Past: Archaeology and the Image. Blackwell Publishing, Oxford.

Elsom-Cook, M. (2001) Principles of interactive multimedia. McGraw-Hill, London.

Fienup-Riordan, A., A. Rearden, and M. Knecht. (2015) Irr'inarqellriit/Amazing Things: Quinhagak Elders Reflect on Their Past. Alaska Journal of Anthropology, 13(2), pp.37-70.

Hardman, L., Van Rossum, G. and Bulterman, D.C. (1993) Structured multimedia authoring. Proceedings of the first ACM international conference on Multimedia, Anaheim, US, August 1993, pp.283-289. Association for Computing Machinery, New York.

Hillerdal, C. (2017) Integrating the Past in the Present: Archaeology as Part of Living Yup'ik Heritage. In C. Hillerdal, A. Karlström, and C-G. Ojala (eds.), Archaeologies of "Us" and "Them": Debating History, Heritage and Indigeneity. Routledge, London and New York.

Hillerdal. C. (2018) Words Apart: Archaeology, indigenous communities and the power of definition. In A. Ekblom, C. Isendahl, and K-J. Lindholm (eds.), The Resilience of heritage. Cultivating a future of the past. Essays in honour of Professor Paul J.J. Sinclair. Uppsala University, Uppsala. 
Hillerdal, C., Knecht, R. and Jones, W. (2019) Nunalleq: Archaeology, climate change and community engagement in a Yup'ik village. Arctic Anthropology, 56(1), pp.4-17.

Huggett, J. (2004) Archaeology and the New Technological Fetishism. Archeologia e Calcolatori, 15, pp.81-92.

Jeffrey, S. (2015) Challenging Heritage Visualisation: Beauty, Aura and Democratisation. Challenging Digital Archaeology, Open Archaeology, 1, pp.144-152.

Kawagley, O. (1990) Yup'ik Ways of Knowing. Canadian Journal of Native Education, 17(2), pp.517.

Law, J. (2004) After Method: Mess in Social Science Research. Routledge, Oxon.

Levy, R. and Dawson, P. (2014) Interactive Worlds as Educational Tools for Understanding Arctic Life. In K, Kee. (ed.), Pastplay: Teaching and Learning History with Technology. University of Michigan Press, Chicago.

Schlag, J. (2018) Surviving the colonial blizzard: The Alaskan Native game Never Alone as a walkthrough in cultural resistance. In $\mathrm{K}$. $\mathrm{N}$. McDaniel (ed.), Virtual Dark Tourism. Ghost Roads. Cham, Palgrave Macmillan, Switzerland.

Serious Animation (2017) SERF Digital Hillforts. www.seriousanimation.com/hillforts (retrieved 7 March 2020)

Serious Animation (2019) Nunalleq: Stories from the Village of Our Ancestors. www.seriousanimation.com/nunalleg (retrieved 7 March 2020).
Richards, C. (2005) Dwelling Among the Monuments. McDonald Institute for Archaeological Research, Cambridge.

Royce, W.W., (1970) Managing the development of large software systems. Proceedings of IEEE WESCON, Los Angeles, US, 4-6 August 1970, pp.328-388. IEEE, New York.

Smith, L. \& Campbell, G. (2016) The Elephant in the Room: heritage, affect and emotion. In W. Logan, M. N. Craith \& U. Kockel, (eds.), A Companion to Heritage Studies, John Wiley \& Sons, Hoboken, NJ.

Smith, L. T. (2012) Decolonizing Methodologies: Research and Indigenous Peoples. Zed Books, London.

Turkle, S. (2009) Simulation and its Discontents. MIT Press, Cambridge, MA.

Watterson, A. (2015) Beyond Digital Dwelling: ReThinking Interpretive Visualisation in Archaeology. Challenging Digital Archaeology, Open Archaeology, 1, pp.119-130.

Watterson, A. and Hillerdal, C. (In review) Nunalleq, Stories from the Village of Our Ancestors: Co-designing a multi-vocal educational resource based on an archaeological excavation. Archaeologies Journal.

Zubrow, E. B. W. (2006) Digital Archaeology: A Historical Context. In Evans, T. L. and Daly, P. (eds.) Digital Archaeology: Bridging Method and Theory. Routledge, New York. 